\title{
A conditional density theorem for the zeros of the Riemann zeta-function
}

\author{
by \\ Alessandro Zaccagnini (Parma)
}

1. Introduction. Let $\varrho:=\beta+i \gamma$ denote the generic non-trivial zero of the Riemann $\zeta$-function,

$$
N(\sigma, T):=\mid\{\varrho=\beta+i \gamma: \beta \geq \sigma,|\gamma| \leq T \text { and } \zeta(\varrho)=0\} \mid
$$

and

$$
\Theta:=\sup \{\beta \leq 1: \zeta(\beta+i \gamma)=0\} .
$$

Our aim is to study the relation between bounds for $N(\sigma, T)$ and bounds for the Selberg integral

$$
J(x, \theta):=\int_{x}^{2 x}|\psi(t)-\psi(t-\theta t)-\theta t|^{2} d t .
$$

It is well known, and comparatively easy to prove, that if $\Theta<1$, then

$$
J(x, \theta) \ll x(\theta x)^{2 \Theta}(\log x)^{B},
$$

for some absolute constant $B$, uniformly for $x^{\varepsilon-1} \leq \theta \leq 1$, for any fixed $\varepsilon>0$ (we have assumed the Density Hypothesis, for simplicity). On the Riemann Hypothesis (i.e., $\Theta=1 / 2$ ), (1) holds with $B=2$ and uniformly for $x^{-1} \leq \theta \leq 1$ (see e.g. Saffari \& Vaughan [6], Lemma 6). Also, it is known that (1) holds unconditionally with $\Theta=1$ and any fixed $B>-2$ in the range $x^{-5 / 6-\varepsilon(x)} \leq \theta \leq 1$, provided that $\varepsilon(x) \rightarrow 0$ as $x \rightarrow \infty$ (see Zaccagnini [9]). It is also well known that any bound of the type

$$
N(\sigma, T) \ll T^{C(1-\sigma)}(\log T)^{B}
$$

for $T \geq 2$, coupled with a suitable zero-free region for the Riemann zetafunction, leads to

$$
J(x, \theta)=o\left(x^{3} \theta^{2}\right)
$$

2000 Mathematics Subject Classification: 11M06, 11M26. 
uniformly for $x^{-2 / C+\varepsilon} \leq \theta \leq 1$, for any fixed $\varepsilon>0$ (see [6], Lemma 5). The best known value for $C$ is $12 / 5$ (Huxley [4]).

We want to prove a sort of converse to the above results, and we study consequences of bounds for $J(x, \theta)$. We show below that a very strong bound for $J(x, \theta)$ like $(1)$ (even if only for $\theta=1$ ) is essentially equivalent to the quasi Riemann Hypothesis, in the sense that $J(x, 1) \ll x^{\delta}$ for some $\delta \in[2,3]$ implies that $\Theta \leq \frac{1}{2}(\delta-1)$. Hence we can confine our attention to the case where the saving over the trivial estimate $J(x, \theta) \ll x^{3} \theta^{2}$, stemming from the Brun-Titchmarsh inequality, is comparatively small. As may be expected from the foregoing discussion, our results will depend both on the strength of the bound for $J$ and on the degree of uniformity in $\theta$ we have. In order to fix notation, we assume that

$$
J(x, \theta) \ll \frac{x^{3} \theta^{2}}{F(\theta x)}
$$

uniformly for

$$
G(x)^{-1} \leq \theta \leq 1,
$$

where $F$ and $G$ are positive, strictly increasing functions, unbounded as $x$ tends to infinity. Our main result is the following

TheOREM. There exist absolute constants $B_{0} \geq 2$ and $C_{0} \geq 1$ such that if (3) holds uniformly in the range (4) for $G(x)=x^{\beta}$, with a fixed $\beta \in(0,1]$, then for any $B \geq \max \left(B_{0}, \beta^{-1}\right)$ and any $C>C_{0}$ we have

$$
N(\sigma, T) \ll_{B, C} \frac{T^{B C(1-\sigma)}}{\min \left(F\left(T^{B-1}\right), T\right)} .
$$

We remark that, since we can assume that $F(x) \ll x^{\varepsilon}$ for every $\varepsilon>0$, in the above hypotheses the denominator is $F\left(T^{B-1}\right)$. Actually, we can give this result a more general form (see (13) below), which gives interesting results also in the case $G(x)=o_{\varepsilon}\left(x^{\varepsilon}\right)$ for every $\varepsilon>0$; for this, see Corollary 2. Some admissible numerical values for $B_{0}$ and $C_{0}$ will be obtained below. We now examine some consequences of the Theorem; the general philosophy is that good estimates for $J$ yield good zero-free regions for zeta.

COROllary 1. If (3) holds for some function $F$ with $F(x) \ll x^{\varepsilon}$ for every $\varepsilon>0$ and $G(x)=x^{\beta}$ for some $\beta \in(0,1]$, then for every $B>B_{0}$ and $t>2$ the Riemann zeta-function has no zeros in the region

$$
\sigma>1-\frac{B-1}{B C_{0}} \cdot \frac{\log F(t)}{\log t} .
$$

The general version referred to above also yields the following special result. 
Corollary 2. Let $B_{0}$ and $C_{0}$ denote the constants in the Theorem. If (3) and (4) hold with $F(x)=\exp (\log x)^{\alpha}$ and $G(x)=\exp (\log x)^{\beta}$ for some fixed $\alpha, \beta \in(0,1]$, then the Riemann zeta-function has no zeros in the region

$$
\sigma>1-\frac{1+o(1)}{B_{0} C_{0}(\log (2+|t|))^{r(\alpha, \beta)}},
$$

where $r(\alpha, \beta):=(1-\min (\alpha, \beta)) \beta^{-1}$.

It should be observed that if, for example, $F(x)=(\log x)^{A}$ then from Corollary 1 we simply recover Littlewood's zero-free region, which is needed in the proof, while arguing as in the proof of Corollary 2 we can show that one recovers the Korobov-Vinogradov zero-free region from (13), provided that one can take $F(x)=G(x)=\exp \left((\log x)^{3 / 5}(\log \log x)^{-1 / 5}\right)$. We also remark that if $F$ were bounded (that is, if we had only the trivial bound for $J$ ) this is essentially Bombieri's proof of his Density Theorem (see Theorem 14 in $[2])$.

The main defect of our Theorem, apart from the fact that $B_{0}$ and $C_{0}$ are very large, is that the range of uniformity (4) cannot be too small in order to exploit the full force of (3). Essentially, our Theorem yields better results if $G(x) \geq F(x)$. This is due to the use of the Brun-Titchmarsh inequality as a universal upper bound for $\psi(t)-\psi(t-\theta t)$ outside the range (4), as in the proof of Lemmas 5 and 6.

Some improvement on the values of the constants is possible, provided one can avoid the use of the Brun-Titchmarsh inequality when estimating the error terms arising from Gallagher's lemma and elsewhere (see Lemma 6). Actually, the numerical values that we obtain are rather large, since the proof yields that the above results hold for some $B_{0} \leq 40000$ and $C_{0} \leq$ $2000 \log 16 e$. This means, for example, that using our main result, even a very strong bound like (3) with $F(x)=x /(\log x)^{2}$ (which is known to hold under RH for $x^{\varepsilon-1} \leq \theta \leq 1$ ) does not lead to a strong Density Theorem, unless $\beta$ is rather large, and, indeed, we need an alternative argument in order to show the complete equivalence with $\mathrm{RH}$.

It will be clear from the proof how the upper bounds for $B_{0}$ and $C_{0}$ depend on other number-theoretic constants. We note that it would be possible in principle to improve on the numerical values given above, though we do not pursue this matter further. It is not difficult to see that a density bound like ours implies estimates for $J(x, \theta)$ like (3), although weaker.

For the proof we use the Second Main Theorem of Turán (Theorem 8.1 of Turán [8]), modifying Bombieri's proof of his Density Theorem (Theorem 14 of [2]). The fact that the zeta-function is of finite order in the critical strip also plays a crucial rôle. These results should be viewed in the light of the papers of Bazzanella \& Perelli [1] and Goldston \& Montgomery [3]. 
It is a pleasure to record my thanks to Jerzy Kaczorowski and Alberto Perelli for several conversations on these topics.

2. Preliminary lemmas. We start with the remark alluded to above, concerning strong bounds for $J(x, 1)$ : assume that

$$
J(x, 1) \ll x^{\delta}
$$

for some $\delta \in[2,3]$. Then $\Theta \leq \frac{1}{2}(\delta-1)$. In fact, writing $\Delta(x):=\psi(x)-x$, one has the well known integral representation

$$
-\frac{\zeta^{\prime}}{\zeta}(s)=\frac{s}{s-1}+s \int_{1}^{\infty} \frac{\Delta(x)}{x^{s+1}} d x=\frac{s}{s-1}+s H(s),
$$

say, in $\sigma>1$, to begin with. But by the Cauchy inequality

$$
|H(s)| \leq \int_{1}^{\infty} \frac{|\Delta(x)|}{x^{\sigma+1}} d x \leq\left\{\int_{1}^{\infty} \frac{|\Delta(x)|^{2}}{x^{\alpha}} d x \int_{1}^{\infty} \frac{d x}{x^{2 \sigma+2-\alpha}}\right\}^{1 / 2} .
$$

By $(5)$, splitting the half line $[1, \infty)$ into the dyadic intervals $\left[2^{n}, 2^{n+1}\right]$, it is easily seen that the first integral converges if $\alpha>\delta$, while the second integral converges absolutely if $\alpha<2 \sigma+1$. If $\sigma>\frac{1}{2}(\delta-1)$ we can find $\alpha$ satisfying both conditions, and hence $H(s)$ represents an analytic function in $\sigma>\frac{1}{2}(\delta-1)$. By $(6)$, the only singularity of $\zeta^{\prime} / \zeta$ in this half plane can occur at $\sigma=1$, and therefore $\zeta$ cannot vanish.

We prove or quote from the literature some results we need later, in order to fix notation. For brevity we write $\mathcal{L}:=\log T$. We write our inequalities with explicit, absolute constants (unless stated otherwise). Hence, from now on $c_{j}$ will denote a positive, absolute constant. We assume throughout that $T$ is sufficiently large, $2 \leq|t| \leq T$ and write $w:=1+i t$.

Lemma 1. There exists an absolute constant $c_{0}>0$ such that for $\mathcal{L}^{-1} \leq$ $r \leq 1 / 4$ the Riemann zeta-function has $\leq c_{0} r \mathcal{L}$ zeros in the circle $|s-w|$ $\leq r$.

This follows from the "Lemme de Densité" in $\S 6$ of [2].

Lemma 2. There exists an absolute constant $c_{1}>0$ such that if $z_{n} \in \mathbb{C}$ for $n=1, \ldots, N$ and $K \in \mathbb{N}$, then there exists $k \in \mathbb{N}$ with $K+1 \leq k \leq K+N$ such that

$$
\left|z_{1}^{k}+\ldots+z_{N}^{k}\right| \geq 2\left(\frac{c_{1} N}{K+N}\right)^{N}\left|z_{1}\right|^{k}
$$

This is a corollary of the Second Main Theorem of Turán (Theorem 8.1 of [8]). Kolesnik \& Straus [5] give the numerical value $c_{1}=(4 e)^{-1}$. Now let

$$
F(s):=-\zeta(s)-\frac{\zeta^{\prime}(s)}{\zeta(s)} .
$$


Lemma 3. Let $c_{1}$ be the constant in Lemma 2. There exists an absolute constant $c_{2}>0$ with the following property: let $\mathcal{L}^{-1} \leq r \leq c_{1} /(16 e)$ and $K \geq c_{2} r \mathcal{L}$. If the Riemann zeta-function has a zero in the circle $|s-w| \leq r$, then there exists an integer $k$ such that $K \leq k \leq 2 K$ and

$$
\frac{1}{k !}\left|F^{(k)}(w+r)\right| \geq \frac{2}{c_{1}}\left(\frac{c_{1}}{4 r}\right)^{k+1} .
$$

Proof. It is well known (see e.g. Titchmarsh [7], Theorem 9.6A) that

$$
\frac{\zeta^{\prime}(s)}{\zeta(s)}=\sum_{|\varrho-w| \leq 1} \frac{1}{s-\varrho}+R_{1}(\mathcal{L})
$$

with $\left|R_{1}(\mathcal{L})\right| \leq c_{3} \mathcal{L}$, in $|s-w| \leq 1 / 2$. Let $\lambda:=A r$ for some constant $A$ to be chosen later, and $s_{0}:=w+r$. We assume that $K$ is an integer satisfying $K \geq c_{2} r \mathcal{L}$, where $c_{2}$ will be chosen eventually. Following the proof of Lemma $\mathrm{A}$ in $\S 6$ of Bombieri [2], we prove that there exists an absolute constant $c_{4}>0$ such that if the Riemann zeta-function has a zero in the circle $|s-w| \leq r$, then for a suitable integer $k \in[K, K+N-1]$, we have

$$
\frac{1}{k !}\left|\left(\frac{d}{d s}\right)^{k} \frac{\zeta^{\prime}}{\zeta}\left(s_{0}\right)\right| \geq 2\left(\frac{c_{1} N}{K+N}\right)^{N}(2 r)^{-k-1}-c_{4} \lambda^{-k} \mathcal{L},
$$

provided that $\lambda \leq 1 / 4$. For the proof, which gives also the bound $c_{4} \leq$ $4 c_{0}+c_{3}$, we need Lemma 1, Lemma 2, (7) and the Cauchy inequalities for the derivatives of holomorphic functions. For $y>0$ define

$$
M(y):=\max _{\left|s-s_{0}\right| \leq y}|\zeta(s)|,
$$

so that, by the Cauchy inequalities again, we have

$$
\frac{1}{k !}\left|\zeta^{(k)}\left(s_{0}\right)\right| \leq(B r)^{-k} M(B r)
$$

for any fixed $B>0$. We remark that the simpler inequality $\left|\zeta^{(k)}\left(s_{0}\right)\right| \ll$ $k ! r^{-k-1}$ would not suffice for our purposes. By Theorem 3.5 of [7] and the Phragmén-Lindelöf principle there exists $c_{5}>0$ such that

$$
|\zeta(\sigma+i t)| \leq c_{5} \cdot \begin{cases}|t|^{c_{6}(1-\sigma)} \log |t| & \text { for } \sigma \in[1 / 2,1] \text { and }|t| \geq 2, \\ \log |t| & \text { for } \sigma \geq 1 \text { and }|t| \geq 2,\end{cases}
$$

where $c_{6}$ is any constant $>2 \mu(1 / 2), \mu$ being the order function (as a Dirichlet series) for the Riemann $\zeta$-function (see [7], Chapter 5). This and (9) yield at once

$$
\frac{1}{k !}\left|\zeta^{(k)}\left(s_{0}\right)\right| \leq c_{5}(B r)^{-k} T^{c_{6}(B-1) r} \mathcal{L}=\frac{4 c_{5}}{c_{1}}\left(\frac{c_{1}}{4 r}\right)^{k+1}\left(\frac{4}{c_{1} B}\right)^{k} T^{c_{6}(B-1) r} r \mathcal{L} .
$$


But for $B \geq 4 c_{1}^{-1}$ we have

$$
\left(\frac{4}{c_{1} B}\right)^{k} \leq\left(\frac{4}{c_{1} B}\right)^{K} \leq\left(\frac{4}{c_{1} B}\right)^{c_{2} r \mathcal{L}}
$$

and

(11) $\frac{1}{k !}\left|\zeta^{(k)}\left(s_{0}\right)\right| \leq \frac{4 c_{5}}{c_{1}}\left(\frac{c_{1}}{4 r}\right)^{k+1} \exp \left\{\left(c_{6}(B-1)-c_{2} \log \frac{c_{1} B}{4}\right) r \mathcal{L}\right\} r \mathcal{L}$.

Using (10) with $B$ replaced by $A$ we prove that, if $A \geq 4 c_{1}^{-1}$, we have

$$
c_{4} \lambda^{-k} \mathcal{L} \leq \frac{4 c_{4}}{c_{1}}\left(\frac{c_{1}}{4 r}\right)^{k+1} \exp \left\{-c_{2} \log \frac{c_{1} A}{4} r \mathcal{L}\right\} r \mathcal{L} .
$$

We finally remark that

$$
\left(\frac{c_{1} N}{K+N}\right)^{N} \geq\left(\frac{c_{1}}{2}\right)^{K} \geq\left(\frac{c_{1}}{2}\right)^{k}
$$

for all $K \geq N$ and $k \geq K$, since $c_{1} \leq 2 e^{-1}$. Hence, by (8), the proof is complete if we show that the sum of the right hand sides of (11) and (12) is $\leq 2 c_{1}^{-1}\left(c_{1} /(4 r)\right)^{k+1}$. This is easily accomplished choosing first $A=B=$ $4 e c_{1}^{-1}$ and then $c_{2}$ large, using the fact that $r \mathcal{L} \geq 1$.

Lemma 4. There exist absolute constants $A_{0} \geq 1, B_{0} \geq 1$ and $C_{0} \geq 2$ with the following property. Let $\mathcal{L}^{-1} \leq r \leq c_{1} /(16 e)$. If the zeta-function has a zero in the circle $|s-w| \leq r$, then for all $x \geq T^{B_{0}}$ and $C>C_{0}$ we have

$$
\int_{x}^{x^{A_{0}}}\left|\sum_{n \in[x, y]} \frac{\Lambda(n)-1}{n^{w}}\right|^{2} \frac{d y}{y} \gg_{C}(\log x)^{3} x^{-C r} .
$$

Pr o of. This is proved essentially as Lemma B in $\S 6$ of [2], using Lemma 3 above. We just remark that the proof gives $B_{0} \geq c_{2}$, the latter being the constant in Lemma 3 , and that $A_{0}=40000$ and $C_{0}=4000 \log \left(4 c_{1}^{-1}\right)$ are admissible values.

LEMMA 5. Uniformly for $x^{\varepsilon-1} \leq \theta \leq 1 / 2$ we have

$$
\int_{x}^{2 x}\left|\sum_{n \in(t-\theta t, t]} \frac{\Lambda(n)-1}{n}\right|^{2} \frac{d t}{t} \ll_{\varepsilon} x^{-3} J(x, \theta)+\theta^{4} .
$$

Proof. For brevity we write $\mathcal{I}:=(t-\theta t, t]$. First we observe that

$$
\sum_{n \in \mathcal{I}} \frac{\Lambda(n)-1}{n}=\sum_{n \in \mathcal{I}} \frac{\Lambda(n)-1}{t}+\sum_{n \in \mathcal{I}}(\Lambda(n)-1)\left(\frac{1}{n}-\frac{1}{t}\right)=\Sigma_{1}+\Sigma_{2},
$$


say. But by the Brun-Titchmarsh inequality we have

$$
\Sigma_{2} \ll \frac{\theta t}{t(t-\theta t)} \sum_{n \in \mathcal{I}}(\Lambda(n)+1) \ll_{\varepsilon} \theta^{2} .
$$

Finally, since $|a+b|^{2} \ll|a|^{2}+|b|^{2}$, we have

$$
\int_{x}^{2 x}\left|\Sigma_{1}+\Sigma_{2}\right|^{2} \frac{d t}{t} \ll_{\varepsilon} \int_{x}^{2 x}\left|\Sigma_{1}\right|^{2} \frac{d t}{t}+\theta^{4} \ll_{\varepsilon} x^{-3} J(x, \theta)+\theta^{4} .
$$

Lemma 6. For $\tau=\exp \theta$ we have

$$
\int_{-\theta^{-1}}^{\theta^{-1}}\left|\sum_{n \in(x, y]} \frac{\Lambda(n)-1}{n} n^{i u}\right|^{2} d u \ll \theta^{-2} \int_{x}^{y}\left|\sum_{n \in(u, \tau u]} \frac{\Lambda(n)-1}{n}\right|^{2} \frac{d u}{u}+\theta .
$$

Proof. We use Gallagher's Lemma (in the form given in [2], Théorème 9), putting

$$
a_{n}:= \begin{cases}\frac{\Lambda(n)-1}{n} & \text { if } n \in(x, y] \\ 0 & \text { otherwise. }\end{cases}
$$

This gives the first term at once. The other term arises from the ranges $(x-\theta x, x)$ and $(y-\theta y, y)$, where we use the Brun-Titchmarsh inequality.

3. Conclusion of the proof. We follow the proof of Theorem 14 in $\S 6$ of Bombieri [2], replacing the large sieve by Lemma 6 with $T:=\theta^{-1}$. Let $\varrho:=\beta+i \gamma$ be a non-trivial zero of $\zeta$, with $\beta>\sigma \geq 1 / 2$. We take $r:=c_{7}(1-\sigma)$ with $c_{7} \geq 2$ and remark that $\mathcal{L}^{-1} \leq r \leq c_{1} /(16 e)$ provided that $1-c_{1} /\left(16 e c_{7}\right) \leq \sigma \leq 1-\left(c_{7} \mathcal{L}\right)^{-1}$. The latter inequality obviously follows from Littlewood's zero-free region if $T$ is large enough. We have

$$
|\varrho-(1+i t)| \leq 1-\beta+|t-\gamma| \leq \frac{1}{2} r+|t-\gamma| \leq r
$$

for $|\gamma-t| \leq r / 2$. From Lemma 4 we have

$$
\int_{x}^{x^{A_{0}}} \int_{\gamma-r / 2}^{\gamma+r / 2}\left|\sum_{n \in(x, y]} \frac{\Lambda(n)-1}{n^{1+i v}}\right|^{2} d v \frac{d y}{y} \gg r(\log x)^{3} x^{-C r},
$$

for any $C>C_{0}$, and, summing over zeros,

$$
N(\sigma, T) r(\log x)^{3} x^{-C r} \ll r \log T \int_{x}^{x^{A_{0}}} \int_{-T-r}^{T+r}\left|\sum_{n \in(x, y]} \frac{\Lambda(n)-1}{n^{1+i u}}\right|^{2} d u \frac{d y}{y},
$$

since each point of the interval $(-T-r, T+r)$ belongs to at most $c_{0} r \mathcal{L}$ 
intervals of type $(\gamma-r / 2, \gamma+r / 2)$, by Lemma 1 . Hence

$$
\begin{aligned}
N(\sigma, T) & \ll \frac{\log T}{(\log x)^{3}} x^{C r} \int_{x}^{x^{A_{0}}} \int_{-T-r}^{T+r}\left|\sum_{n \in(x, y]} \frac{\Lambda(n)-1}{n^{1+i u}}\right|^{2} d u \frac{d y}{y} \\
& \ll \frac{\log T}{(\log x)^{3}} x^{C r}\left\{\theta^{-2} \int_{x}^{x_{0}} \int_{x}^{y}\left|\sum_{n \in(u, \tau u]} \frac{\Lambda(n)-1}{n}\right|^{2} \frac{d u}{u} \frac{d y}{y}+\theta \log x\right\},
\end{aligned}
$$

by Lemma 6 with $T=\theta^{-1}, \tau=\exp \theta$. The inner integral is

$$
\begin{aligned}
& \ll \log x \max _{x \leq t \leq y} \int_{t}^{2 t}\left|\sum_{n \in(u, \tau u]} \frac{\Lambda(n)-1}{n}\right|^{2} \frac{d u}{u} \\
& \ll \log x \max _{x \leq t \leq y}\left\{J(t, \tau-1) t^{-3}+\theta^{4}\right\},
\end{aligned}
$$

by Lemma 5. By our hypothesis (3) and our choice of $T$, we finally have

$$
\begin{aligned}
N(\sigma, T) & \ll \frac{\log T}{\log x} x^{C r}\left\{\frac{1}{F(\theta x)}+\frac{\theta}{\log x}+\theta^{2}\right\} \\
& \ll \frac{\log T}{\log x} x^{C r}\left\{\frac{1}{F\left(x T^{-1}\right)}+T^{-1}\right\} .
\end{aligned}
$$

This is our main estimate, subject to the conditions $x \geq \max \left(T^{B_{0}}, G^{-1}(T)\right)$ and $\theta=T^{-1}$, where $G^{-1}$ denotes the inverse function of $G$.

The proof of Theorem 1 is completed by choosing $x=T^{B}$, with $B \geq B_{0}$ and $B \geq \beta^{-1}$.

Proof of Corollary 2. If $\alpha>\beta$ then our hypotheses imply that the rightmost expression in curly brackets in (13) is $\ll T^{-1}$. We choose $x$ as small as possible (that is, $\left.x=\exp (\log T)^{1 / \beta}\right)$ and obtain the result. If, instead, $\alpha \leq \beta$, the expression considered above is $\ll F\left(x T^{-1}\right)$, and a simple computation, again with $x=\exp (\log T)^{1 / \beta}$, gives the zero-free region

$$
\sigma>1-\frac{(\log T)^{\alpha / \beta}(1+o(1))}{C(\log T)^{1 / \beta}} .
$$

This concludes the proof.

\section{References}

[1] D. Bazzanella and A. Perelli, The exceptional set for the number of primes in short intervals, to appear.

[2] E. Bombieri, Le grand crible dans la théorie analytique des nombres, Astérisque 18 (1974). 
[3] D. A. Goldston and H. L. Montgomery, Pair correlation of zeros and primes in short intervals, in: Analytic Number Theory and Diophantine Problems, A. Adolphson et al. (eds.), Birkhäuser, Boston, 1987, 183-203.

[4] M. N. Huxley, On the difference between consecutive primes, Invent. Math. 15 (1972), 164-170.

[5] G. Kolesnik and E. G. Straus, On the sum of powers of complex numbers, in: Studies in Pure Mathematics, To the Memory of P. Turán, P. Erdős (ed.), Birkhäuser, Basel, 1983, 427-442.

[6] B. Saffari and R. C. Vaughan, On the fractional parts of $x / n$ and related sequences. II, Ann. Inst. Fourier (Grenoble) 27 (1977), no. 2, 1-30.

[7] E. C. Titchmarsh, The Theory of the Riemann Zeta-Function, 2nd ed., Oxford Univ. Press, 1986.

[8] P. Turán, On a New Method of Analysis and its Applications, Wiley, New York, 1984.

[9] A. Zaccagnini, Primes in almost all short intervals, Acta Arith. 84 (1998), 225-244.

Dipartimento di Matematica

Università degli Studi di Parma

via Massimo d'Azeglio 85/A

43100 Parma, Italy

E-mail: zaccagni@prmat.math.unipr.it

URL: http://www.math.unipr.it/ zaccagni/home.html

Received on 29.3.1999

and in revised form on 22.9.1999 